\title{
Comparison of Brain Activation in Response to Two Dimensional and Three Dimensional On-Line Games
}

\author{
Woo Hyun Song ${ }^{1}$, Doug Hyun $\operatorname{Han}^{1 凶}$ and Hyung Jin Shim ${ }^{2}$ \\ ${ }^{1}$ Departments of Psychiatry and ${ }^{2}$ Radiology, Chung-Ang University Hospital, Seoul, Republic of Korea
}

\begin{abstract}
Objective The present study assessed the difference in the brain activity of professional gamers (excessive players, but not addicts) in response to playing a 3-dimensional online game with an improved interface.

Methods Twenty-three StarCraft I pro gamers and 16 StarCraft II pro gamers were recruited at Chung Ang University Medical Center. Brain activity in response to StarCraft I or II cues was assessed with a 1.5 Tesla Espree MRI scanner.

Results StarCraft I pro gamers showed significantly greater activity in 4 clusters in response to the video game cues compared to StarCraft II pro gamers: right superior frontal gyrus, right medial frontal gyrus, right occipital lobe, and left medial frontal gyrus. StarCraft II pro gamers showed significantly greater activity in 3 clusters in response to the video game cues compared to StarCraft I pro gamers: left middle frontal gyrus, left temporal fusiform gyrus and left cerebellum.

Discussion This is the first study to show the difference in brain activity between gamers playing either a 2-dimensional or 3-dimensional online game. Current brain imaging studies may confirm the pro gamers' experience when playing StarCraft II, a 3-dimensional game with an improved interface, relative to playing StarCraft I.

Psychiatry Investig 2013;10:115-120
\end{abstract}

Key Words StarCraft, Three dimensional, Two dimensional, Pro-gamer, Functional magnetic resonance imaging.

\section{INTRODUCTION}

\section{StarCraft I vs. StarCraft II}

StarCraft is one of the bestselling real-time strategy online games. More than 11 million copies of StarCraft I have been sold worldwide since 1998. StartCraft II: Wings of Liberty was released in 2010. Three different races, the Zerg, the Protoss, and the Terrans, perform differently using various tactics with the purpose of allowing a player to defeat their opponent. ${ }^{1}$ StarCraft I and StarCraft II share basic formats of game play while using the same characters. However, there are 2 main differences between StarCraft I and StarCraft II. ${ }^{2,3}$ First, StarCraft II is a 3-dimensional game, while StarCraft I is a 2-dimensional game. Second, StarCraft II is somewhat easier for players to control due to improvements in the interface over

Received: October 8, 2012 Revised: November 7, 2012

Accepted: November 20, 2012 Available online: May 30, 2013

$\triangle$ Correspondence: Doug Hyun Han, MD, PhD

Department of Psychiatry, Chung-Ang University Hospital, 102 Heukseok-ro, Seoul 156-755, Republic of Korea

Tel: +82-2-6299-3132, Fax: +82-2-6299-1114, E-mail: hduk@yahoo.com

(a) This is an Open Access article distributed under the terms of the Creative Commons Attribution Non-Commercial License (http://creativecommons.org/licenses/bync/3.0) which permits unrestricted non-commercial use, distribution, and reproduction in any medium, provided the original work is properly cited.

\section{StarCraft I. ${ }^{2}$}

In South Korea, professional gamers (pro gamers) are players who are characterized by extensive online game playing, but who do not meet the criteria for game addiction. These players are not more impulsive or depressed compared with general users. ${ }^{4}$ Like other professional sports players, pro gamers have contracts or an annual salary and belong to teams. They maintain a regular lifestyle and are dedicated to their profession despite challenging competition. To become a pro gamer, candidates must complete a two-step competition: they must win a competition including 300 volunteers and a competition involving 8 to 10 semi-pro gamers. ${ }^{5}$ There are currently approximately 200 StarCraft I pro gamers on 8 StarCraft I teams and approximately 100 StarCraft II pro gamers on 9 StarCraft II teams in Korea. ${ }^{5}$

\section{2 dimensions (2D) vs. 3 dimensions (3D)}

One of the important visual functions is to assess the 3-dimensional shape of objects in response to first encountering that object. ${ }^{6}$ Studies of $3 \mathrm{D}$ perception have suggested that this perception may be systemically distorted. ${ }^{7,8}$ For example, a straight line in the environment can be perceived as a curved line, and apparent intervals in depth become systemically 
compressed with increased viewing distance. In addition, studies of the perceptual representation of 3D shapes have suggested that many factors and cognitive interpretations, including depth and orientation, object surface, occlusion contour, surface color, and line-drawings of a simple plane-faced polyhedra can affect 3D perception. ${ }^{9,10}$ Recent studies have attempted to assess brain activity in response to various factors affecting perception. ${ }^{11,12}$ In the assessment of event-related potential in response to the surface difference between typical and atypical color objects, the N350 time window was found in 26 healthy subjects. ${ }^{11}$ The result indicates that surface color is an important cue in the facilitation of long-term memory in $3 \mathrm{D}$ perception. In a $\mathrm{fMRI}$ study of $3 \mathrm{D}$ perception, activity in the bilateral parieto-temporal junction and dorsal portion of the right precuneus was shown to increase in response to increased movement of a $3 \mathrm{D}$ ball induced by a cast shadow. ${ }^{12}$

\section{Game play and interface}

Recent studies have suggested that video game training could alter cognitive function and brain activity. ${ }^{13,14}$ HubertWallander et al. ${ }^{14}$ demonstrated that action video games could enhance selective attention and the spatial distribution of vision. Lee et al. ${ }^{13}$ reported that 30 th video game (Fortress ${ }^{\circledR}$ ) training could alter cognitive functions, including visuospatial attention, goal-directed motor planning, and activity of the right dorsolateral prefrontal cortex, in healthy subjects.

Computer video games are thought of as goal-driven interactive entertainment which includes challenges and conflicts which results in pleasure and intellectually engaging simulation. ${ }^{15,16}$ In the human brain, the anterior cingulate and dorsolateral prefrontal corticies are well-known to be associated with conflict control. ${ }^{17}$

There are significant improvements in terms of interface in StarCraft II, compared to StarCraft I. ${ }^{18}$ First, the movements of worker 'AI' in StarCraft II are conducted automatically to increase the effectiveness of gathering the mineral while the movements of worker 'SCV' in StarCraft I should manually be controlled by gamers. In addition, StarCraft II needs more multi-tasking abilities to make the strategy with improved rally system, multi-selecting buildings, and pin-point mouse control. For this reason, Many pro gamers reported that StarCraft I needed more multi-tasking functions and finger movements to improve the interface relative to StarCraft II. ${ }^{2}$

\section{Hypothesis}

The goal of the present study was to assess differences in brain activity in response to playing a 3D online game with an improved interface in pro gamers who were not addicts, but only excessive players. The authors hypothesized that the activity of prefrontal cortex and temporal cortex would be dif- ferent between the pro gamers playing StarCraft I (2D) and StarCraft II (3D).

\section{METHODS}

\section{Subjects}

Twenty-three StarCraft I pro gamers and 16 StarCraft II pro gamers were recruited at Chung Ang University Medical Center. All subjects were screened using the Structured Clinical Interview for DSM-IV and the Beck Depression Inventory (BDI). ${ }^{19}$ Exclusion criteria for both groups were: 1) BDI scores $>19$; 2) other axis I psychiatric disorders including substance abuse; 3) head injury or trauma history; or 4) impaired behaviors or distress due to maladaptive patterns of online game playing. The research protocol was approved by the Chung Ang University Hospital Institutional Review Board. Written informed consent was provided by all participants. All gamers were male. There were no significant differences in age $(\mathrm{z}=0.37, \mathrm{p}=0.71)$, years of education $(\mathrm{z}=0.8, \mathrm{p}=0.42)$, time spent playing games $(\mathrm{z}=0.21, \mathrm{p}=0.84)$, professional gaming career length $(\mathrm{z}=1.59, \mathrm{p}=0.11)$, Young's Internet Addiction Scale (YIAS) $(\mathrm{z}=0.72, \mathrm{p}=0.47)$, BDI scores $(\mathrm{z}=1.16, \mathrm{p}=0.25)$, alcohol consumption $\left(\chi^{2}=0.45, \mathrm{p}=0.5\right)$ or smoking $\left(\chi^{2}=0.12\right.$, $\mathrm{p}=0.73$ ) between StarCraft I and StarCraft II pro gamers (Table 1).

The age of the StarCraft I pro gamers was $20.5 \pm 1.6$ years, their education year was $12.2 \pm 0.6$ years, and their career length was $2.5 \pm 1.31$ years. StarCraft I pro gamers played StarCraft I an average of $10.0 \pm 1.6$ hours/day. The mean YIAS score of StarCraft I pro gamers was $37.7 \pm 10.5$ and their mean BDI score was $6.5 \pm 4.3$. The age of the StarCraft II pro gamers was $20.4 \pm 2.1$ years, their education level was $12.1 \pm 1.1$ years, and their career length was $1.8 \pm 0.4$ years. StarCraft II pro

Table 1. Demographic characteristics of subjects

\begin{tabular}{lccc}
\hline & $\begin{array}{c}\text { StarCraft I } \\
(23)\end{array}$ & $\begin{array}{c}\text { StarCraft II } \\
(16)\end{array}$ & Statistics \\
\hline Sex (male/female) & $23 / 0$ & $16 / 0$ & \\
Age & $20.5 \pm 1.6$ & $20.4 \pm 2.1$ & $\mathrm{Z}=0.37, \mathrm{p}=0.71$ \\
Education (year) & $12.2 \pm 0.6$ & $12.1 \pm 1.1$ & $\mathrm{Z}=0.8, \mathrm{p}=0.42$ \\
Game time & $10.0 \pm 1.6$ & $9.9 \pm 1.6$ & $\mathrm{Z}=0.21, \mathrm{p}=0.84$ \\
Career (year) & $2.5 \pm 1.31$ & $1.8 \pm 0.4$ & $\mathrm{Z}=1.59, \mathrm{p}=0.11$ \\
YIAS & $37.7 \pm 10.5$ & $39.3 \pm 9.5$ & $\mathrm{Z}=0.72, \mathrm{p}=0.47$ \\
BDI & $6.5 \pm 4.3$ & $7.6 \pm 4.2$ & $\mathrm{Z}=1.16, \mathrm{p}=0.25$ \\
Alcohol & $9 / 14$ & $8 / 8$ & $\chi^{2}=0.45, \mathrm{p}=0.5$ \\
$\quad($ drink/non-drink) & & & \\
Smoking (smoker/ & $6 / 18$ & $5 / 11$ & $\chi^{2}=0.12, \mathrm{p}=0.73$ \\
non-smoker) & & & \\
\hline YIAS: Youngs Intem & & & \\
\hline
\end{tabular}

YIAS: Young's Internet Addiction Scale, BDI: Beck Depression Inventory 
gamers played StarCraft II an average of $9.9 \pm 1.6$ hours/day. The mean YIAS score of StarCraft II pro gamers was $39.3 \pm 9.5$ and their mean BDI score was 7.6 \pm 4.2 .

\section{Study procedure}

Assessment of brain activity and craving for game play

All MR imaging was performed on a 1.5-Tesla Espree MRI scanner (SIEMENS, Erlangen, Germany). The subjects were shown a 450-second silent recording consisting of five continuous 90-second segments. Each 90-second segment consisted of three 30-second sub-segments. The 90-second segments consisted of a white cross on a black background (B), a neutral control ( $\mathrm{N}$, humanoid robot animation scenes), and the video game cue (C, "StarCraft I" or "StarCraft II" scene). The 5 segments were ordered as follows: B-N-C, B-C-N, C-B$\mathrm{N}, \mathrm{N}-\mathrm{B}-\mathrm{C}$, and C-N-B. The video was presented using an IFIS-SA ${ }^{\mathrm{TM}}$ system (MRI Device Corporation, Waukesha, WI, USA) over a single fMRI scanning session. The fMRI session was recorded using a gradient-recalled echo planar imaging (EPI) sequence [37 transverse slices, $5.0 \mathrm{~mm}$ thickness, a voxel size of $3.5 \times 3.5 \times 5.0 \mathrm{~mm}, \mathrm{TE}=30 \mathrm{~ms}, \mathrm{TR}=3000 \mathrm{~ms}$, in-plane resolution $=64 \times 64$ pixels, field of view $(\mathrm{FOV})=230 \times 230 \mathrm{~mm}$ ] at 3-second intervals. For anatomical imaging, 3D T1-weighted magnetization-prepared rapid gradient echo (MPRAGE) data were collected with the following parameters: $T R=1500$ $\mathrm{ms}, \mathrm{TE}=3.00 \mathrm{~ms}, \mathrm{FOV}=256 \times 256 \mathrm{~mm}, 128$ slices, $1.0 \times 1.0 \times$ $1.33 \mathrm{~mm}$ voxel size.

\section{fMRI data analysis}

Brain activity in current research was analyzed using the Brain Voyager software package (BVQX 1.9, Brain Innovation, Maastricht, Netherlands). The fMRI time series for each subject was co-registered to the anatomical 3-dimensional data set using the multi-scale algorithm provided by BVQX. The structural images were spatially normalized to standard Talairach space. The same nonlinear transformation was applied to the $\mathrm{T} 2{ }^{*}$-weighted fMRI time series data. After slice scan time correction and 3D motion correction, the functional data were spatially smoothed using Gaussian kernel with an FWHM of $6 \mathrm{~mm}$ and temporally smoothed using a Gaussian kernel of $4 \mathrm{~s}$ using algorithms provided by BVQX.

\section{Statistical analysis}

The model functions were used as explanatory variables within the context of the general linear model (GLM) to apply multiple linear regression analysis to fMRI signal timecourses on a voxel-by-voxel basis. A random effects analysis yielded individual and group statistical parametric maps of brain activation contrasting the video game cue vs. neutral sti- muli. For all analyses, associations were regarded as significant if the False Discovery Rate (FDR) was less than or equal to 0.01 (corrected for multiple comparisons) in 100 adjacent voxels. A second-level analysis of random effects ANOVA model with two within-subject factors (video game cue vs. neutral stimuli) and two between-subject factors (StarCraft I and StarCraft II) was used to show the differences in brain activation between StarCraft I and StarCraft II pro gamers.

\section{RESULTS}

\section{StarCraft I or StarCraft II stimulation vs. neutral control}

In responding to StarCraft I stimuli compared to neutral stimuli, StarCraft I pro gamers showed significantly greater activity in two clusters (FDR $<0.01, \mathrm{p}<0.0006$ ): right middle frontal gyrus [Talairach $\mathrm{x}, \mathrm{y}, \mathrm{z} ; 56,-35,23$; Brodmann area (BA) 6] and right occipital lobe (2, -64, 21; BA 7)(Table 2, Figure 1). In responding to StarCraft II stimuli compared to neutral stimuli, StarCraft II pro gamers showed significantly greater activity in three clusters (FDR $<0.01, \mathrm{p}<0.0007$ ): right superior frontal gyrus (Talairach $\mathrm{x}, \mathrm{y}, \mathrm{z} ; 26,7,54$; BA 6), right parietal lobe (2, -66, 35; BA 7), and left middle frontal gyrus $(-33,0,54$; BA 6)(Table 2, Figure 1).

\section{StarCraft I pro gamers vs. StarCraft II pro gamers}

Compared to StarCraft II pro gamers, StarCraft I pro gamers showed significantly greater activity in four clusters in response to a video game cue ( $F D R<0.01, \mathrm{p}<0.0005)$ : right superior frontal gyrus $(53,-39,19$; BA 13), right medial frontal gyrus $(7,54,3$; BA 10), right occipital lobe (2, -74, 16; BA 18), and left medial frontal gyrus (-11, 54, 4; BA 10)(Table 2, Figure 1).

Compared to StarCraft I pro gamers, StarCraft II pro gamers showed significantly greater activity in three clusters in response to a video game cue (FDR $<0.01, \mathrm{p}<0.0005)$ : left middle frontal gyrus $(-32,2,51$; BA 6$)$, left temporal fusiform gyrus (-42, -46, -24; BA 36), and left cerebellum $(-42,-51,-30)$ (Table 2, Figure 1).

\section{DISCUSSION}

This is the first study to examine the differences in brain activity between players of $2 \mathrm{D}$ and $3 \mathrm{D}$ games. The two games studied share the same plot and similar characters, and the participants are not addicts, only excessive players. The current brain imaging study may support the sense of StarCraft II pro-gamers in terms of three dimension and improved interface relative to StarCraft I. 
Table 2. Brain regions in response to StarCraft I vs. StarCraft II

\begin{tabular}{|c|c|c|c|c|c|}
\hline $\mathrm{x}$ & $\mathrm{y}$ & $\mathrm{z}$ & Voxels & $\mathrm{p}$ values & Regions \\
\hline \multicolumn{6}{|c|}{ StarCraft 1 pro-gamers } \\
\hline 22 & -10 & 49 & 100 & $\mathrm{FDR}_{<0.01}, \mathrm{p}=0.0006$ & Right middle frontal gyrus, BA 6 \\
\hline 2 & -64 & 32 & 700 & $\mathrm{FDR}_{<0.01}, \mathrm{p}=0.0006$ & Right occipital lobe, cuneus, BA 7 \\
\hline \multicolumn{6}{|c|}{ StarCraft 2 pro-gamers } \\
\hline 26 & 7 & 54 & 250 & $\mathrm{FDR}_{<0.01}, \mathrm{p}=0.0007$ & Right superior frontal gyrus, BA 6 \\
\hline 2 & -66 & 35 & 1800 & $\mathrm{FDR}_{<0.01}, \mathrm{p}=0.0007$ & Right parietal lobe, precuneus, BA 7 \\
\hline-33 & 0 & 54 & 100 & $\mathrm{FDR}_{<0.01}, \mathrm{p}=0.0007$ & Left middle frontal gyrus, BA 6 \\
\hline \multicolumn{6}{|c|}{ StarCraft 1 pro-gamers $>$ StarCraft 2 pro-gamers } \\
\hline 53 & -39 & 19 & 240 & $\mathrm{FDR}_{<0.01}, \mathrm{p}=0.0005$ & Right superior temporal gyrus, BA 13 \\
\hline 7 & 54 & 3 & 120 & $\mathrm{FDR}_{<0.01}, \mathrm{p}=0.0005$ & Right medial frontal gyrus, BA 10 \\
\hline 2 & -74 & 16 & 600 & $\mathrm{FDR}_{<0.01}, \mathrm{p}=0.0005$ & Right occipital lobe, cuneus, BA 18 \\
\hline-11 & 54 & 4 & 100 & $\mathrm{FDR}_{<0.01}, \mathrm{p}=0.0005$ & Left medial frontal gyrus, BA 10 \\
\hline \multicolumn{6}{|c|}{ StarCraft 1 pro-gamers $<$ StarCraft 2 pro-gamers } \\
\hline-32 & 2 & 51 & 150 & $\mathrm{FDR}_{<0.01}, \mathrm{p}=0.0005$ & Left middle frontal gyrus, BA 6 \\
\hline-42 & -46 & -24 & 150 & $\mathrm{FDR}_{<0.01}, \mathrm{p}=0.0005$ & Left temporal, fusiform gyrus, BA 36 \\
\hline-42 & -51 & -30 & 150 & $\mathrm{FDR}_{<0.01}, \mathrm{p}=0.0005$ & Left cerebellum, anterior lobe, culmen \\
\hline
\end{tabular}

BA: Brodmann area, FDR: False Discovery Rate

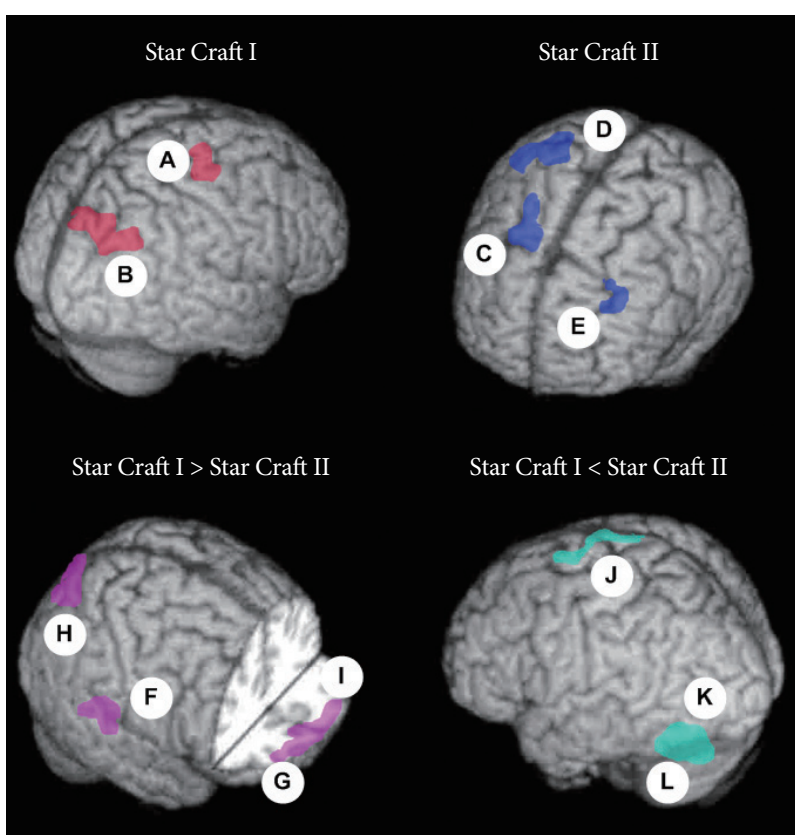

Figure 1. Brain regions in response to StarCraft I vs. StarCraft II. A: Right middle frontal gyrus, BA 6. B: Right occipital lobe, cuneus, BA 7. C: Right superior frontal gyrus, BA 6. D: Right parietal lobe, precuneus, BA 7. E: Left middle frontal gyrus, BA 6. F: Right superior temporal gyrus, BA 13. G: Right medial frontal gyrus, BA 10. H: Right occipital lobe, cuneus, BA 18. I: Left medial frontal gyrus, BA 10. J: Left middle frontal gyrus, BA 6. K: Left temporal, fusiform gyrus, BA 36. L: Left cerebellum, anterior lobe, culmen.

\section{Brain response to online game playing in pro gamers}

Compared to previous studies examining general game players or patients with game addiction, ${ }^{20,21}$ the brain activity in both StarCraft I and II pro gamers was focused on the frontal cortex. In 20 healthy university students, the left inferior frontal gyrus, left parahippocampal gyrus, right and left parietal lobe, right and left thalamus, and right cerebellum were activated in response to game cues. ${ }^{20}$ Ko et al. ${ }^{21}$ reported that the right orbitofrontal cortex, right nucleus accumbens, right and left anterior cingulate and medial frontal cortex, right dorsolateral prefrontal cortex, and right caudate nucleus were activated in the patients with game addiction in response to game cues. In the comparison of brain volume, pro gamers showed increased gray matter volume in the left cingulate gyrus compared to non-gamer subjects. ${ }^{4}$

\section{D vs. 3D}

Compared to StarCraft I pro gamers, StarCraft II pro gamers showed increased activity in the back left side of the brain, including the left temporal fusiform gyrus and left cerebellum. In response to 3D stimulation using random dot stereograms, the activity of the back left side of the brain was increased..$^{22}$ Recent studies have suggested that inter-individual differences in 3D surface perception might be associated with brain activity in the superior temporal area. ${ }^{23-29}$ In a subdural electrode recording, Gonzalez et al..$^{25}$ reported the basal temporo-parietal cortex, including the fusiform gyrus, had a crucial role in high-level stereoscopic information processing. Some of these findings are also consistent with the results obtained using electrophysiological recordings of single neurons within the dorsal and ventral pathways of monkeys. Janssen et al. ${ }^{26-29}$ have shown that neurons in the temporal area are selective for disparity-defined 3D shapes and that this selectivity 
arises from processing of the global 3D structure of the shape. In PET studies, temporal fusiform gyrus plays a role in perception, representation and memorization of shapes in the construction of 3D-representationsof objects. ${ }^{30}$

In addition, several fMRI studies have suggested that the areas around the lateral occipital and parieto-occipital cortices are associated with stereoscopic processing. ${ }^{31-35}$ Georgieva et al. ${ }^{34}$ suggested that the extraction of 3D shape from textures involves the bilateral caudal inferior temporal gyrus, lateral occipital sulcus and several bilateral sites along the intraparietal sulcus. Gerardin et al. ${ }^{35}$ suggested that activations in higher occipito-temporal and parietal areas predict better the perceived 3D shape irrespective of the light direction. In addition, the posterior fusiform gyrus is thought to act as an interface between visual information and other meaningful stimulus properties including associated sound and meaning. ${ }^{36}$ Kober and Neuper ${ }^{37}$ also reported that three dimensional view of virtual reality system increased the cortical activation of parietal lobe with the evidence of increased alpha band in EEG.

Sex and mental training were thought to be associated the performance and brain activation during two- vs. three-dimensional presentation. ${ }^{38}$ Especially, men may rely more on left hemisphere processing than women in response to rotational tasks. ${ }^{39}$ The subjects in current research were male progamers and the differences of brain activity between StarCraft I and II was left side. Further studies should be focused in female gamers and training courses.

\section{Interface}

Compared to StarCraft II pro gamers, StarCraft I pro gamers showed increased activity in both sides of the medial frontal gyrus (orbitofrontal cortex). However, StarCraft II pro gamers showed increased activity in the left middle frontal gyrus (dorsolateral prefrontal cortex, DLPFC) compared to StarCraft I pro gamers.

The right inferior frontal gyrus (orbitofrontal cortex) has been reported to be implicated in go/no-go tasks. ${ }^{40}$ In particular, the activity of the inferior frontal gyrus increased in response to an aversion condition (e.g., the subject stops pressing a button when a red signal appears) during go/no-go tasks. ${ }^{41}$ In a study using the Stroop conflict test, the medial frontal cortex was thought to be involved in the interaction between pre-response conflict and inhibition of return, whereas the left DLPFC was involved in the interaction between response conflict and inhibition of return. ${ }^{17}$ In a fMRI study, Goldberg et al. ${ }^{42}$ found that the superior frontal gyrus (DLPFC) was involved in self-awareness (self-related observer function), in coordination with the action of the sensory system. This could be explained as an inhibition signal to accept a risky option. Increased activity in the DLPFC in StarCraft II pro gamers may be correlated with the improved interface in Starcraft II. Many pro gamers commented that StarCraft II pro gamers paid more attention to strategy and game play, while StarCraft I pro gamers were more concerned with the dull artificial intelligence and finger speed required for pressing the keyboard and mouse buttons during game play due to the inferior interface. ${ }^{2}$

\section{Limitations}

There were several limitations to the current study. First, the assessment of StarCraft I and II in the current study was somewhat arbitrary since it depended on the pro gamers' subjective experiences and newspaper articles. Future studies should be conducted in order to provide confirmation of the results of the present study. Second, because the game used in the current research was limited to StarCraft, caution is necessary regarding generalization to other pro gamers.

\section{Conclusions}

The increased activity in the left side of the basal temporal area, including the fusiform gyrus, may be associated with the 3D presentation of StarCraft II. In addition, use of the inferior interface of StarCraft I may be associated with activation of the orbitofrontal cortex.

\section{Acknowledgments}

This work was supported by Korean Game Culture Foundation.

\section{REFERENCES}

1. McNeil G. StarCraft: I, Mengsk. New York, NY: A division of Simon \& Schuster, Inc; 2012.

2. Nam YS. Improved Interface of Star Craft II. Seoul: Daily e Sports; 2012. http://esports.dailygame.co.kr/news/read.php?id=60899.

3. Tony H. Starcraft 2 vs Starcraft 1 - What's Different? Michigan: Enzinearticle; 2010. http://EzineArticles.com/3885787.

4. Han DH, Lyoo IK, Renshaw PF. Differential regional gray matter volumes in patients with on-line game addiction and professional gamers. J Psychiatr Res 2012;46:507-515.

5. Team and Players of Korea eSport Association (AeSPA). Available at: http://www.e-sports.or.kr.

6. Todd JT. The visual perception of 3D shape. Trends Cogn Sci 2004;8: 115-121.

7. Koenderink JJ, van Doorn AJ, Lappin JS. Direct measurement of the curvature of visual space. Perception 2000;29:69-79.

8. Todd JT, Norman JF. The visual perception of 3-D shape from multiple cues: are observers capable of perceiving metric structure? Percept Psychophys 2003;65:31-47.

9. Huffman DA. Realizable configurations of lines in pictures of polyhedra. Machine Intelligence 1977;8:493-509.

10. Singh M, Hoffman DD. Completing visual contours: the relationship between relatability and minimizing inflections. Percept Psychophys 1999;61:943-951.

11. Bramao I, Faisca L, Forkstam C, Inacio F, Araujo S, Petersson KM, et al. The interaction between surface color and color knowledge: behavioral and electrophysiological evidence. Brain Cogn 2012;78:28-37.

12. Katsuyama N, Usui N, Nose I, Taira M. Perception of object motion in three-dimensional space induced by cast shadows. Neuroimage 2011; 
54:485-494.

13. Lee H, Voss MW, Prakash RS, Boot WR, Vo LT, Basak C, et al. Videogame training strategy-induced change in brain function during a complex visuomotor task. Behav Brain Res 2012;232:348-357.

14. Hubert-Wallander B, Green CS, Sugarman M, Bavelier D. Changes in search rate but not in the dynamics of exogenous attention in action videogame players. Atten Percept Psychophys 2011;73:2399-2412.

15. Bethke E. Game Development and Production. Texas: Wordware Publish Inc; 2003.

16. Checkland P, Scholes J. Soft Systems Methodology in Action. New Jersey: John Wiley and Sons Ltd; 1999.

17. Chen Q, Wei P, Zhou X. Distinct neural correlates for resolving stroop conflict at inhibited and noninhibited locations in inhibition of return. J Cogn Neurosci 2006;18:1937-1946.

18. Interface Changes and Their Effect on Gameplay. Available at: http:// www.gamereplays.org/starcraft $2 /$ portals.php? show=page $\&$ name $=$ starcraft_2_interface_blizzcon_preview. Accessed November 5, 2012.

19. Beck AT, Ward CH, Mendelson M, Mock J, Erbaugh J. An inventory for measuring depression. Arch Gen Psychiatry 1961;4:561-571.

20. Han DH, Bolo N, Daniels MA, Arenella L, Lyoo IK, Renshaw PF. Brain activity and desire for Internet video game play. Compr Psychiatry 2011;52:88-95.

21. Ko CH, Liu GC, Hsiao S, Yen JY, Yang MJ, Lin WC, et al. Brain activities associated with gaming urge of online gaming addiction. J Psychiatr Res 2009;43:739-747.

22. Hagura H, Nakajima M. Study of asthenopia caused by the viewing of stereoscopic images: measurement by MEG and other devices. Proc SPIE 2006;6057:6057ok.

23. Beer AL, Roder B. Attending to visual or auditory motion affects perception within and across modalities: an event-related potential study. Eur J Neurosci 2005;21:1116-1130.

24. Beer AL, Watanabe T, Ni R, Sasaki Y, Andersen GJ. 3D surface perception from motion involves a temporal-parietal network. Eur J Neurosci 2009;30:703-713.

25. Gonzalez F, Relova JL, Prieto A, Peleteiro M. Evidence of basal temporo-occipital cortex involvement in stereoscopic vision in humans: a study with subdural electrode recordings. Cereb Cortex 2005;15:117122.

26. Janssen P, Vogels R, Liu Y, Orban GA. Macaque inferior temporal neurons are selective for three-dimensional boundaries and surfaces. J Neurosci 2001;21:9419-9429.

27. Janssen P, Vogels R, Orban GA. Macaque inferior temporal neurons are selective for disparity-defined three-dimensional shapes. Proc Natl Acad
Sci U S A 1999;96:8217-8222.

28. Janssen P, Vogels R, Orban GA. Selectivity for 3D shape that reveals distinct areas within macaque inferior temporal cortex. Science 2000; 288(5473):2054-2056.

29. Janssen P, Vogels R, Orban GA. Three-dimensional shape coding in inferior temporal cortex. Neuron 2000;27:385-397.

30. Faillenot I, Sakata H, Costes N, Decety J, Jeannerod M. Visual working memory for shape and 3D-orientation: a PET study. Neuroreport 1997; 8:859-862.

31. Iwami T, Nishida Y, Hayashi O, Kimura M, Sakai M, Kani K, et al. Common neural processing regions for dynamic and static stereopsis in human parieto-occipital cortices. Neurosci Lett 2002;327:29-32.

32. Kwee IL, Fujii Y, Matsuzawa H, Nakada T. Perceptual processing of stereopsis in humans: high-field (3.0-tesla) functional MRI study. Neurology 1999;53:1599-1601.

33. Peuskens H, Claeys KG, Todd JT, Norman JF, Van Hecke P, Orban GA. Attention to 3-D shape, 3-D motion, and texture in 3-D structure from motion displays. J Cogn Neurosci 2004;16:665-682.

34. Georgieva SS, Todd JT, Peeters R, Orban GA. The extraction of 3D shape from texture and shading in the human brain. Cereb Cortex 2008; 18:2416-2438.

35. Gerardin P, Kourtzi Z, Mamassian P. Prior knowledge of illumination for 3D perception in the human brain. Proc Natl Acad Sci U S A 2010; 107:16309-16314.

36. Devlin JT, Jamison HL, Gonnerman LM, Matthews PM. The role of the posterior fusiform gyrus in reading. J Cogn Neurosci 2006;18:911-922.

37. Kober SE, Neuper C. Sex differences in human EEG theta oscillations during spatial navigation in virtual reality. Int J Psychophysiol 2011;79: 347-355.

38. Neubauer AC, Bergner S, Schatz M. Two- vs. three-dimensional presentation of mental rotation tasks: Sex differences and effects of training on performance and brain activation. Intelligence 2010;38:529-539.

39. Parsons TD, Larson P, Kratz K, Thiebaux M, Bluestein B, Buckwalter JG, et al. Sex differences in mental rotation and spatial rotation in a virtual environment. Neuropsychologia 2004;42:555-562.

40. Aron AR, Robbins TW, Poldrack RA. Inhibition and the right inferior frontal cortex. Trends Cogn Sci 2004;8:170-177.

41. Christopoulos GI, Tobler PN, Bossaerts P, Dolan RJ, Schultz W. Neural correlates of value, risk, and risk aversion contributing to decision making under risk. J Neurosci 2009;29:12574-12583.

42. Goldberg II, Harel M, Malach R. When the brain loses its self: prefrontal inactivation during sensorimotor processing. Neuron 2006;50:329339. 